\title{
Antenna effects in terahertz apertureless near-field optical microscopy
}

\author{
Kanglin Wang and Daniel M. Mittleman \\ Department of Electrical and Computer Engineering, MS 366, Rice University, Houston, \\ Texas 77251-1892 \\ Nick C. J. van der Valk and Paul C. M. Planken ${ }^{\text {a) }}$ \\ University of Technology Delft, Faculty of Applied Sciences, Department of Imaging Science \\ and Technology, Lorentzweg 1, 2628 CJ Delft, the Netherlands
}

(Received 14 November 2003; accepted 4 August 2004)

\begin{abstract}
We have performed measurements on terahertz $(\mathrm{THz})$ apertureless near-field microscopy that show that the temporal shape of the observed near-field signals is approximately proportional to the time-integral of the incident field. Associated with this signal change is a bandwidth reduction by approximately a factor of 3 which is observed using both a near-field detection technique and a far-field detection technique. Using a dipole antenna model, it is shown how the observed effects can be explained by the signal filtering properties of the metal tips used in the experiments. (C) 2004 American Institute of Physics. [DOI: 10.1063/1.1797554]
\end{abstract}

Apertureless near-field scanning optical microscopy (ANSOM) is an attractive technique for obtaining subwavelength resolution in optical imaging at visible and midinfared wavelengths. ${ }^{1-3}$ In this technique, light is scattered off a subwavelength-sized metal tip which is held close to a surface. The scattered light is collected in the far-field, giving subwavelength resolution in the immediate neighborhood of the tip apex. Very recently, there have been reports showing how ANSOM can be adapted for the terahertz (THz) frequency domain, by combining it with $\mathrm{THz}$ time-domain spectroscopy. ${ }^{4-7}$ This is an exciting development, as it is becoming increasingly clear that many systems of interest, including biological molecules and a variety of artificial nanostructures, have absorption features in the $\mathrm{THz}$ frequency range, but are far smaller than the wavelength of the $\mathrm{THz}$ radiation. ${ }^{8-11}$ These developments therefore offer great promise for $\mathrm{THz}$ microscopy. In the THz ANSOM experiments, two different detection techniques are currently employed: THz electric fields are either detected in the nearfield of the tip using electro-optic sampling, or in the far-field of the tip with a receiver. In the latter case, near-field information from the tip apex is obtained by vibrating the metal tip at a high frequency, followed by phase-sensitive detection at this frequency. However, an important aspect of both experiments remains unexplained: The measured temporal profile of the near-field signals is very different from that of the incident $\mathrm{THz}$ pulses. Associated with this shape change is a bandwidth reduction of about a factor of 3 , which could limit the utility of the technique in spectroscopic measurements. For practical applications, such as $\mathrm{THz}$ microscopy, it is essential that the origin of this bandwidth reduction is understood.

Here, we present measurements and calculations which demonstrate that the observed bandwidth reduction is a consequence of the antenna properties of the metal tips used in the experiments. We demonstrate that, regardless of whether the near-field signal is observed directly under the tip or in the far-field, it is approximately proportional to the timeintegral of the incident $\mathrm{THz}$ signal. Near-field calculations

\footnotetext{
${ }^{a)}$ Electronic mail: p.c.m.planken@tnw.tudelft.nl
}

based on a dipole antenna model qualitatively reproduce all the essential features observed in the measurements and provide insight into ANSOM experiments in general.

The two THz ANSOM configurations, used for the experiments, are schematically shown in Figs. 1(a) and 1(b). In Fig. 1(a) $\mathrm{THz}$ pulses from an ultrafast photoconductive switch are incident on a subwavelength copper-berylium tip of $10 \mu \mathrm{m}$ diameter, which is held close to a metal surface. The scattered $\mathrm{THz}$ radiation, emerging from the tip/sample system, is focused onto a photoconductive, time-gated detector. The tip is vibrated at $750 \mathrm{~Hz}$ and subsequent lock-in detection at this frequency is used to ensure that only signals coming from the near-field of the tip apex are measured. In the setup illustrated in Fig. 1(b), THz pulses are incident on a subwavelength copper tip of $20 \mu \mathrm{m}$ diameter, held close to the surface of a (100) oriented GaP crystal. In this case the tip is not vibrated. THz electric-field polarization components perpendicular to the crystal surface, which develop near the tip apex, are electro-optically detected in the $\mathrm{GaP}$

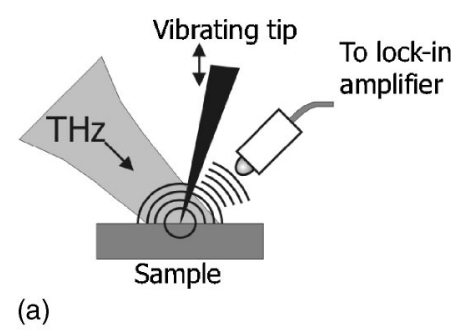

(b)

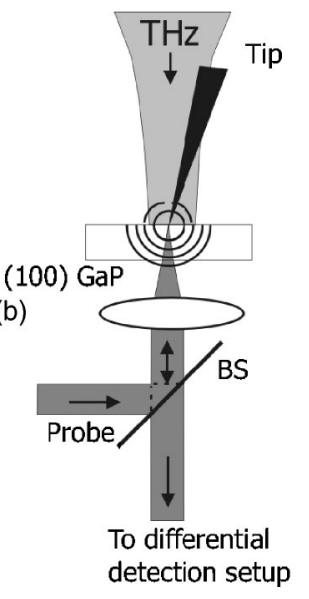

(c)

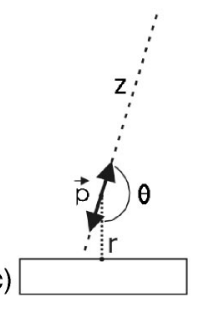

FIG. 1. (a) "Far-field" THz ANSOM setup. The THz pulse is detected in the far-field while the tip is vibrated. (b) "Near-field" THz ANSOM setup. The $\mathrm{THz}$ pulse is detected in the near-field of the tip. (c) Angle and coordinates of the oscillating dipole. 

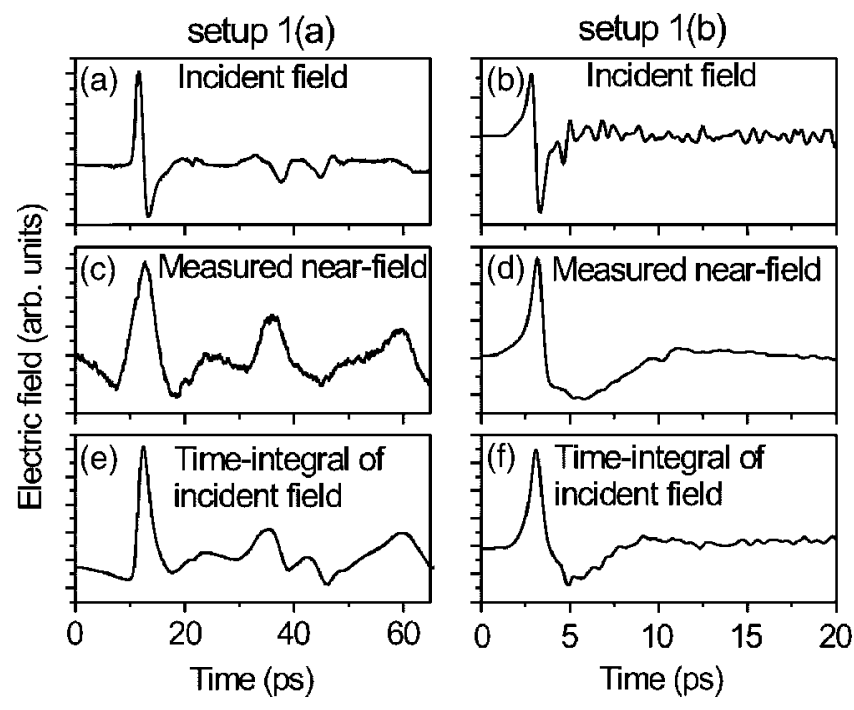

FIG. 2. THz electric fields measured with THz ANSOM setup 1(a) (left) and $\mathrm{THz}$ ANSOM setup 1(b) (right). The top graphs (a) and (b) correspond to measurements of the $\mathrm{THz}$ pulses incident on the metal tip, (c) and (d) correspond to measurements of the near-fields, and (e) and (f) correspond to the calculated time-integral of the incident fields.

crystal by a probe laser pulse, which is focused to a $10 \mu \mathrm{m}$ spot size directly underneath the tip. Details of these setups can be found in Refs. 4-6. The difference between the setups shown in Figs. 1(a) and 1(b) is primarily that in Fig. 1(a) the $\mathrm{THz}$ near-field signal is measured in the far-field whereas in Fig. 1(b) the THz electric field is directly sensed in the nearfield of the tip.

In Figs. 2(a) and 2(b) we plot the measured THz electric fields incident on the metal tips in setups 1(a) and 1(b), respectively. Although the signals look somewhat different, as they are measured in different laboratories, they both consist of approximately a single cycle of the electric field, and contain frequency components in the $\mathrm{THz}$ range. The somewhat broader emission bandwidth of the THz source used in setup 1(b), also gives rise to more pronounced oscillatory signals, visible after the main peak in Fig. 1(b). They are caused by the absorption and re-emission of $\mathrm{THz}$ light by water vapor molecules in the atmosphere.

In Figs. 2(c) and 2(d), we plot the measured near-field $\mathrm{THz}$ signals obtained with setups 1(a) and 1(b), respectively. The results are strikingly different from the incident $\mathrm{THz}$ electric-field transients shown in Figs. 2(a) and 2(b). Instead of a signal consisting of approximately a single cycle of the electric field, we now measure a $\mathrm{THz}$ transient consisting of a positive peak, followed by a relatively slowly varying, negative tail. We note that the results obtained in the two different laboratories are very similar, indicating that the observed effects are quite general and not specific to the detection method. The frequency spectra that correspond with the measured near-field data resemble low-pass filtered versions of the incident spectra (Fig. 3).

From these measurements, an immediate and important conclusion can be drawn: In THz ANSOM experiments, the near-field spectrum cannot be assumed to be the same as the spectrum of the input radiation. A crucial clue regarding the explanation for the difference between the incident and the near-field signals is obtained when we calculate the timeintegral of the incident $\mathrm{THz}$ pulse. The results are plotted in Figs. 2(e) and 2(f). These curves reproduce the essential fea-

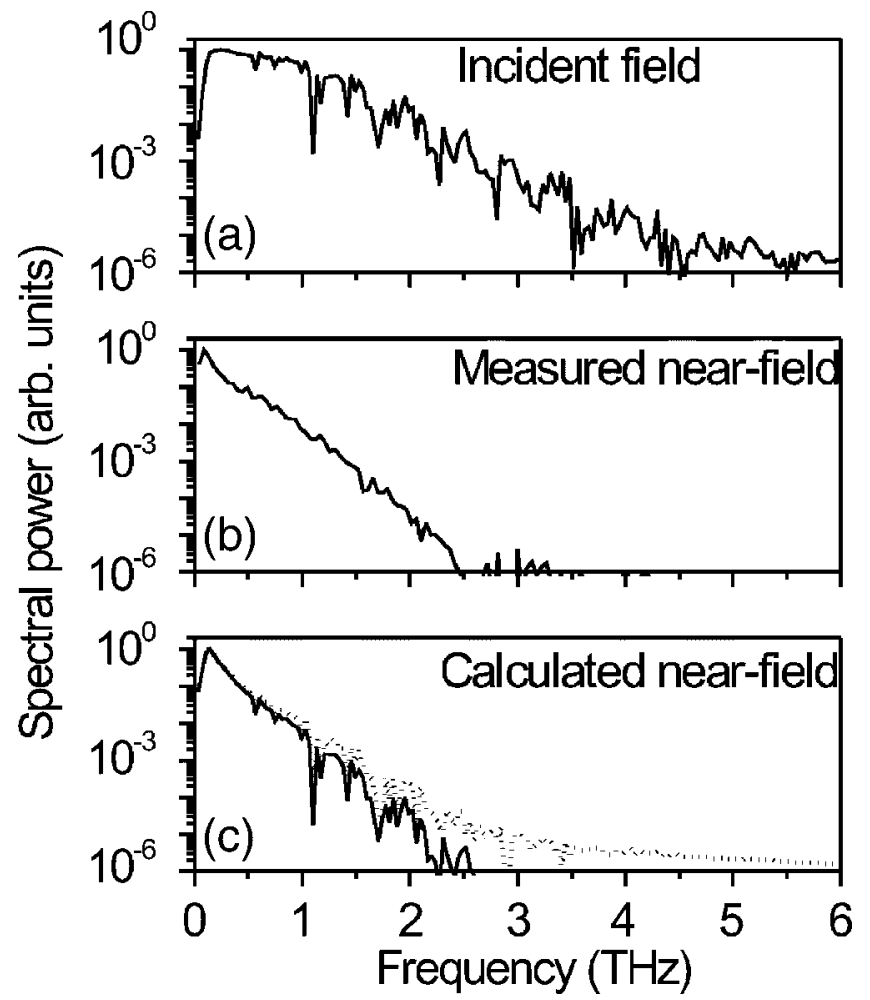

FIG. 3. (a) Power spectrum of the measured incident THz pulses. (b) Power spectrum of the measured $\mathrm{THz}$ near-field pulse, and (c) power spectrum of the calculated $\mathrm{THz}$ near-field, with $R=150 \Omega, C=10^{-13} \mathrm{~F}$, and $L=2$ $\times 10^{-11} \mathrm{H}$ (solid line), and the power spectrum of the near-field calculated using resistive coupling only (dotted line).

tures of the measured near-field transients. We note that integrating in the time-domain is identical to a multiplication by $1 / \omega$ in the frequency domain, so the measured near-field spectra lose much of their high frequency content.

To fully simulate our measurements, detailed numerical calculations would be needed, taking into account Maxwell's equations, the presence of a dielectric medium, and the exact shape of the tip. Physical insight is obtained, however, by employing a simple model, in which we treat the tip as an oscillating dipole. With $z, r$, and $\theta$ defined as in Fig. 1(c), we can write for the electric field emitted by the dipole $\mathbf{p}:^{12}$

$$
\begin{aligned}
\mathbf{E}= & \frac{\mu_{0} c^{2}}{4 \pi}\left[\hat{\boldsymbol{\theta}} \sin \theta\left(\frac{p}{r^{3}}+\frac{p^{\prime}}{c r^{2}}+\frac{p^{\prime \prime}}{c^{2} r}\right)\right. \\
& \left.+\hat{\mathbf{r}} \cos \theta\left(\frac{2 p}{r^{3}}+\frac{2 p^{\prime}}{c r^{2}}\right)\right],
\end{aligned}
$$

where $p$ is the oscillating dipole moment $(p \| z)$ taken to be oriented along the tip, $p^{\prime}$ and $p^{\prime \prime}$ are the first and second time-derivatives of $p$, and $r$ is the distance from the dipole. The $p$ and $p^{\prime}$ terms decrease rapidly with distance from the dipole and are the near-field terms, whereas the $p^{\prime \prime}$ term corresponds to the far-field radiation.

We now concentrate on the setup 1(b) in which we electro-optically measure the $\mathrm{THz}$ near-field component perpendicular to the crystal surface, underneath the tip. At short distances, the $1 / r^{3}$ terms dominate and we can thus write for the component of the electric field perpendicular to the crystal surface,

license or copyright; see http://apl.aip.org/about/rights_and_permissions 


$$
E_{\perp, \mathrm{nf}} \propto \frac{\mu_{0} c^{2}}{4 \pi} \frac{p}{r^{3}},
$$

showing that the measured near-field must be proportional to the dipole moment $p$. We now relate the dipole moment to the incident $\mathrm{THz}$ electric field using an antenna model. The continuity equation gives us a relation between the dipole moment $p$ and the current $I_{\mathrm{THz}}$ in an antenna,

$$
p(t) \propto \int_{-\infty}^{t} I_{\mathrm{THz}}\left(t^{\prime}\right) d t^{\prime},
$$

where, for simplicity, the current is assumed to be the same everywhere along the antenna. ${ }^{13}$ We note that expression (2), which is valid for a point dipole, can also be used in the case of a real antenna, assuming that the field is calculated for a distance away from the antenna, larger than the relevant antenna dimensions. ${ }^{14,15}$ A relation between the current and the incident $\mathrm{THz}$ electric field can be obtained by treating the antenna as a simple electronic network consisting of a radiation resistance $R_{r}$, a capacitor $C$, and an inductance $L$, all in series. The driving "voltage" $V_{\mathrm{THz}}$ of this network is the incident $\mathrm{THz}$ electric field $E_{\mathrm{THz}}$. The relation between this electric field and the induced current $I_{\mathrm{THz}}$ for this network in the frequency domain is

$$
I_{\mathrm{THz}}(\omega) \propto \frac{E_{\mathrm{THz}}(\omega)}{\left(R_{r}+j \omega L-j(\omega C)^{-1}\right)} .
$$

If we assume, for simplicity, that $L=0$, and $R_{r}$ and $C$ are large, then Eq. (4) can be approximated by $I_{\mathrm{THz}}(\omega)$ $\propto E_{\mathrm{THz}}(\omega) / R_{r}$, which is equivalent to $I_{\mathrm{THz}}(t) \propto E_{\mathrm{THz}}(t) / R_{r}$. This is the regime of resistive coupling. Using Eq. (3), this gives us $E_{\perp, \mathrm{nf}} \propto p(t) \propto \int_{-\infty}^{t} E_{\mathrm{THz}}\left(t^{\prime}\right) d t^{\prime}$, showing that the nearfield is proportional to the time-integral of the incident field, as we have seen in Fig. 2. In Fig. 3, we plot the spectrum of the measured incident $\mathrm{THz}$ pulse (top), the spectrum of the measured near-field (middle), and the spectrum of the calculated near-field (bottom), obtained by "filtering" the incident THz field using a $L C R$ network, with certain reasonable values of $R_{r}, C$ and $L$, indicated in the figure caption. The agreement between the calculated and the measured near-field spectra is even better. For comparison, we also plot the calculated THz near-field spectrum assuming resistive coupling only. In this case, there is less agreement between the calculation and the measurement. The difference in spectral power between the two calculations grows with increasing frequency, which is caused by the increasing relative importance of inductive coupling at higher frequencies. We note that by using either much smaller antennas $\left(R_{r}, C\right.$, and $L$ small) or by decreasing the frequency $\omega$, we obtain $I_{\mathrm{THz}}(\omega)$ $\propto j \omega E_{\mathrm{THz}}(\omega)$ and therefore $p(t) \propto E_{\mathrm{THz}}(t)$. This corresponds to the regime of capacitive coupling employed by Knoll et al. in their calculations for midinfrared ANSOM experiments. ${ }^{1,3}$

It may seem suprising that the near-field signal measured with setup 1(a) is also similar to the time-integral of the incident field, as this signal is radiated by the antenna, and not measured in its near-field. One possible explanation for this involves the image dipole induced in the metal substrate. This image dipole generates an additional electric field near the real antenna dipole according to Eq. (2). This field only "sees" a short antenna of fixed length $l$ for which the radiation resistance is proportional to $R_{r} \propto \omega^{2} l^{2}$. ${ }^{14}$ Using Eqs. (3) and (4), and assuming that $R_{r}$ dominates over $L$ and $C$, we deduce that $p(t) \propto \int_{-\infty}^{t} \int_{-\infty}^{t^{\prime}} \int_{-\infty}^{t^{\prime \prime}} E_{\mathrm{THz}}\left(t^{\prime \prime \prime}\right) d t^{\prime \prime \prime} d t^{\prime \prime} d t^{\prime}$. The far-field radiated by this dipole [see Eq. (1)] and measured by setup 1 (a) is proportional to the second time-derivative of $p$ giving $p^{\prime \prime} \propto \int_{-\infty}^{t} E_{\mathrm{THz}}\left(t^{\prime}\right) d t^{\prime}$. Note that for the $\mathrm{THz}$ beam originally incident on the antenna, we assume that $R_{r}$ is constant. This is justified for the focused $\mathrm{THz}$ beam for which the product $\omega l$ of the frequency and the effective antenna length is constant.

In conclusion, we have shown measurements and calculations, which demonstrate that the bandwidth reduction observed in THz ANSOM experiments is caused by the antenna properties of the metal tip employed in these experiments. Our results indicate that the metal tips are both resistively and inductively coupled to the incident $\mathrm{THz}$ radiation, and that inductive coupling increases in significance at higher frequencies. Although our results were obtained at frequencies below $2.5 \mathrm{THz}$, we would like to point out that they have implications for ANSOM experiments at higher frequencies as well.

This work was performed as part of the "Stichting voor Fundamenteel Onderzoek der Materie (FOM)," which is financially supported by the "Nederlandse Organisatie voor Wetenschappelijk Onderzoek (NWO)." This work was also partially supported by the National Science Foundation, and by Advanced Micro Devices.

${ }^{1}$ B. Knoll and F. Keilmann, Opt. Commun. 182, 321 (2000).

${ }^{2}$ L. Aigouy, F. X. Andréani, A. C. Boccara, J. C. Rivoal, J. A. Porto, R. Carminati, J.-J. Greffet, and R. Mégy, Appl. Phys. Lett. 76, 397 (2000).

${ }^{3}$ R. Hillenbrand, T. Taubner, and F. Keilmann, Nature (London) 418, 159 (2002).

${ }^{4}$ N. C. J. van der Valk and P. C. M. Planken, Appl. Phys. Lett. 81, 1558 (2002).

${ }^{5}$ G. Zhao, R. N. Schouten, N. van der Valk, W. Th. Wenckebach, and P. C. M. Planken, Rev. Sci. Instrum. 73, 1715 (2002).

${ }^{6}$ K. Wang, A. Barkan, and D. M. Mittleman, Appl. Phys. Lett. 84, 305 (2004).

${ }^{7}$ H.-T. Chen, R. Kersting, and G. C. Cho, Appl. Phys. Lett. 83, 3009 (2003).

${ }^{8}$ B. M. Fischer, M. Walther, and P. Uhd Jepsen, Phys. Med. Biol. 47, 3807 (2002).

${ }^{9}$ M. Brucherseifer, M. Nagel, P. Haring Bolivar, and H. Kurz, Appl. Phys. Lett. 77, 4049 (2000).

${ }^{10}$ J. E. Boyd, A. Briskman, V. L. Colvin, and D. M. Mittleman, Phys. Rev. Lett. 87, 147401 (2001).

${ }^{11}$ M. C. Beard, G. M. Turner, and C. A. Schmuttenmaer, Nano Lett. 2, 983 (2002).

${ }^{12}$ G. L. Pollack and D. R. Stump, Electromagnetism (Addison-Wesley, San Fransisco, 2002).

${ }^{13}$ In reality the current must be zero at the antenna ends.

${ }^{14}$ J. D. Kraus, Antennas, 2nd ed. (McGraw-Hill, New York, 1988).

${ }^{15}$ We have experimentally verified that the signal shape is unchanged when the tip-crystal distance is decreased from a distance of $100 \mu \mathrm{m}$ (where the model is valid) to a distance of $0 \mu \mathrm{m}$, where strictly speaking, the model is no longer valid. 